\title{
EVALUATING THE ROBUSTNESS OF AN ACTIVE NETWORK MANAGEMENT FUNCTION IN AN OPERATIONAL ENVIRONMENT
}

\author{
Alberto VENTURI* \\ alb11102@strath.ac.uk \\ Paul WRIGHT** \\ paul.wright@npl.co.uk
}

Andrew J. ROSCOE*

andrew.roscoe@eee.strath.ac.uk

\author{
Michael J. DOLAN* \\ michael.dolan@strath.ac.uk \\ Alistair FORBES**
alistair.forbes@npl.co.uk \\ Graham W. AULT* \\ graham.ault@strath.ac.uk
}

\author{
Paul CLARKSON** \\ paul.clarkson@npl.co.uk \\ Xin-She YANG** \\ xin-she.yang@npl.co.uk \\ Graeme M. BURT* \\ graeme.burt@strath.ac.uk
}

* University of Strathclyde, Scotland $\quad * *$ National Physical Laboratory, UK

\begin{abstract}
This paper presents the integration process of a distribution network Active Network Management (ANM) function within an operational environment in the form of a MicroGrid Laboratory. This enables emulation of a real power network and enables investigation into the effects of data uncertainty on an online and automatic ANM algorithm's control decisions. The algorithm implemented within the operational environment is a Power Flow Management (PFM) approach based around the Constraint Satisfaction Problem (CSP). This paper shows the impact of increasing uncertainty, in the input data available for an ANM scheme in terms of the variation in control actions. The inclusion of a State Estimator (SE), with known tolerances is shown to improve the ANM performance.
\end{abstract}

\section{INTRODUCTION}

Automatic control systems, based on software tools, are becoming more desirable in distribution power systems with the proliferation of renewable energy resources. Such schemes are expected to, primarily, manage system voltage fluctuations, network power flows and fault levels. However, other functionality is emerging that includes power balancing, system frequency control and management of demand side resources for the primary system constraints. A critical concern that requires addressing is the robustness of online and automatic ANM algorithms/schemes. The ANM scheme's functionality depends on convergence to a solution when faced with uncertainty and its reliability can be reduced by data skew and error [1]. In addition, very few measurements are currently taken at the distribution level and it is perhaps unrealistic to imagine that voltage and power flow are measured at every node. The number of measurements points will be limited in the first instance through economic reasons: instrumentation and information analysis are expensive and would be limited to the minimum necessary amount to obtain a satisfactory level of visibility of the network conditions.

The work presented within this paper evaluates PFM functionality based on the Constraint Satisfaction Problem (CSP) [2] in an operational environment. The objective is to assess performances when subjected to different levels of data uncertainty and verify the introduction of a SE in the ANM architecture to mitigate the data uncertainty effects on the control action.

\section{PFM USING AN ONLINE CSP APPROACH}

Modelling the PFM problem as a CSP entails expressing the problem as a set of variables with finite discrete domains and a set of constraints. For PFM, the problem to be solved is concerned with deciding what control actions to take, on the Distributed Generation (DG) units, in order to maintain the network within the thermal limits (i.e. constraints). Therefore, the variables of the CSP are the controllable generating plant power output set-points and the domains are discrete values that the generators' set-points can assume. These values are the maximum values that a generator can output (i.e. control signals). However, the intermittent nature of most renewable generating plants means that DG output is such that its output is continuous up to this discrete set-point value. PFM modelled as a CSP can be represented as [3]:

$$
\begin{aligned}
& \qquad\left(\mathrm{V}_{\text {gens }}, \mathrm{D}_{\text {Control Signal }}, \mathrm{C}\right) \\
& \text { Where: } \\
& \mathrm{V}_{\text {gens }}=\left\{\text { Gen }_{1, G \ldots n_{2 \ldots}} \text { Gen }_{\mathrm{n}}\right\} \\
& \mathrm{D}_{\text {Control Signal }} \text { is: } \\
& \mathrm{D}_{\text {Gen } 1}=\{1, \ldots, 0\}, \mathrm{D}_{\text {Gen } 2}=\{1, \ldots, 0\}, \mathrm{D}_{\text {Genn }}=\left\{v_{1}, \ldots, v_{n}\right\}
\end{aligned}
$$

$\mathrm{C}$ is the constraint applied to the sets of variables:

$$
\begin{aligned}
& \mathrm{C}_{\text {Power Flow }}=\left\{\left|S_{i j}\right| \leq S_{i j}^{\max }\right\} \\
& \mathrm{C}_{\text {Contractual }}=\{k, l, m\} \\
& C_{\text {MaxDG }}=\left\{\max \sum_{n=1}^{N} P_{G i}\right\}
\end{aligned}
$$

Where $S_{i j}$ are line power flows associated with the line 
power flow limit, $S_{i j}^{\max } ; k, l$ and $m$ are integer numbers indicating the order in which generators Gen $_{1}$, Gen $_{2}$ and $G_{e n}$ were connected and where $P_{G i}$ is the calculated output of each DG unit.

Modelling PFM, in this way, relies upon a load flow engine to evaluate the power flows within the network to determine any control actions that are required. In addition, it is possible to rank the generators in a last-in, first off (LIFO) manner to replicate the current connection regime used in the UK. One of the characteristics of CSP is that multiple solutions can be returned. This is valuable when measurement errors exist and the first solution does not alleviate the network constraints. The preference constraint would return the solution that curtails the least amount of generation whilst meeting the contractual (i.e. LIFO) and load flow constraints. Figure 1 depicts the inputs and outputs of the CSP PFM approach. Further information regarding this approach can be found in $[2,3]$.

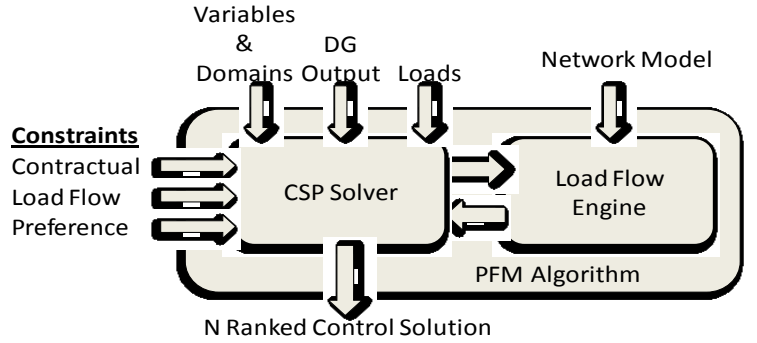

Figure 1 - Inputs and outputs of the CSP PFM algorithm $[2,3]$

\section{A REALISTIC TEST ENVIRONMENT}

Part of the microgrid available at Strathclyde University [4] has been configured to allow the integration and testing process of an ANM function or scheme. The subsection of the microgrid network, used for this purpose, can include a wide variety of loads, generation equipment and measurement sensors that can easily be reconfigured to emulate different future smart grid network scenarios. The present grid set up for these initial ANM tests consist of seven buses and is shown in Figure 2.

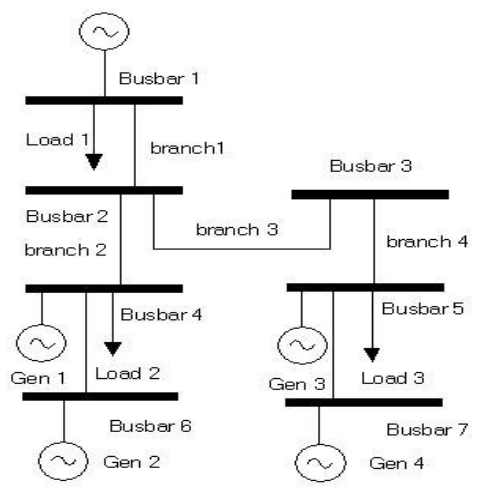

Figure 2 - Microgrid schema

The first bus is connected to the main power supply and includes a variable load bank. Load banks are also connected to two other busbars (4 and 5 in Figure 2). Induction machines, which can also act as generators, are connected to buses $4,5,6$ and 7 . These units have a maximum real power output of $2.2 \mathrm{~kW}, 5.5 \mathrm{~kW}, 7.5 \mathrm{~kW}$ and $7.5 \mathrm{~kW}$, respectively.

The PFM algorithm, presented above, was chosen as the ANM control approach to manage the outputs of the DG units depicted in Figure 2. As well as the power network representing a genuine network, an attempt has been made to configure the measurement data and communication system to represent modern utility practices. The software was installed on a computer (mimicking that of a substation platform) and connected to the microgrid network.

The sensor measurements coming from the microgrid are collected via a real time station (RTS) computer developed by ADI. The RTS has analogue and digital input/output (I/O) interfaces, can execute programs written with Matlab and Simulink, process directly the data collected, manage the electrical machines of the grid and guarantee their safe operation. After a first elaboration, the measurements are then made available, via GUI, on a standard PC. The PFM software was installed on an independent standard Windows PC with the different parts of the system communicating through OPC (Object linking and embedding for Process Control) functionality and using IEC 61850 as the common data model. The data coming from the grid sensors are mapped on the OPC server variables and sent from the PC connected to the RTS to the OPC server. The control software reads them, through the OPC server, and sends the control signals back through the RTS. To overcome the problems originated by the DCOM communication protocol the OpenOPC tool [5], was installed to bypass the DCOM security settings.

Using this approach the PFM software can evaluate the microgrid power flows and can compute and issue control commands when thermal limitations have been reached. These control commands are in the form of DG curtailment set-points as defined by the variables' domain values in the CSP formulation.

To update the RTS with control commands computed by the PFM software (that is to return control signals for implementation) the RTS analogue inputs are used. In order to send the data to the RTS analogue inputs a Beckhoff CX5010 Embedded PC with Intel ${ }^{\circledR}$ Atom ${ }^{\mathrm{TM}}$ processor has been used. The Beckhoff software presents OPC functionalities and the data can be sent using the OPC standard, specifying the correct path. A specific piece of software, based on the OpenOPC functionalities, was written in order to allow communications to these different OPC servers.

Visibility of the ANM software is achieved through a local GUI that shows current DG output information and the implemented control signals along with the information used to arrive at the control signal. That is, the name of the line(s) overloaded, the actual power flow(s), the line 
rating(s), the time of the event and the computed control actions. This GUI is updated each time new data is received and analysed by the PFM software.

\section{EXPERIMENTAL WORK AND RESULTS}

The sensors installed on the microgrid and the data acquisition system guarantee a precision of $2.14 \%$ in the measurement of voltage and current magnitude, and consequently a precision of $4.5 \%$ in measuring the power flow. This level of precision is considered enough to simulate the real operating conditions of an energy management system on a low voltage network. Under real conditions the data coming from the actual networks are taken from a few measurement points, often only current and voltage at the substation are measured.. Any additional data requirements would be substituted with data taken, for example, from load customer profiles (pseudomeasurements). For example in [7] three cases are presented in which the uncertainty of real measurements is assumed to be between $1 \%$ and $3 \%$ and the uncertainty of the pseudomeasurements between $20 \%$ and $50 \%$. The error for real measurements can also be more prevalent for example in the presence of harmonic distortion on the line [8].

In order to show the effect of the uncertainty of the data on the performance of the ANM software a series of tests were executed on the microgrid. The induction machines located at buses 4, 5, 6 and 7 were set to compensate the load requested on the buses 4 and 5 . Thermal constraints were set in the branches 1 and 3 by derating the limits within the PFM software and microgrid network model. The generator access priorities were assigned to represent a LIFO connection arrangement. Gen 1 was set to 1 (i.e. it has the highest priority), Gen 3 was set to 2 , Gen 2 was set to 3 and Gen 4 was set to 4 (i.e. this unit would be the first to be curtailed if a thermal breach was detected). Then, progressively, the loads were reduced to zero starting with the load on busbar 5. This caused a rising power flow through the branches 1 and 3, and a consequent thermal constraint violation.

The response of the ANM function, for this scenario, was evaluated against the following data sets:

- An initial clean set of input data without any uncertainty

- A set of data as collected from the grid (with an uncertainty of $4.5 \%$ )

- A set of data in which the uncertainty of the loads and the machines power flow was artificially increased to $6 \%$

- A set of data as calculated by a state estimator (SE) that reduces the uncertainty to $2 \%$

In Figure 3 the different control signals sent by the ANM to curtail the power output of the generator on busbar 7, Gen 4 , in presence of different levels of uncertainty are shown.
As a result of the steady decrease in loads at busbars 4 and 5 , the ANM reduces regularly the power output of the generators when the data is not affected by any uncertainty. This can be seen in Figure 3 with Gen 4 being curtailed from $50 \%$ output down to zero output (tripped off) over the scenario period. When considering the actual measuring coming from the microgrid $(4.5 \%$ uncertainty), the curtailment is relatively regular (compared to the case with no uncertainty) however the curtailment depth is bigger than that necessary to take in account of the uncertainty. Furthermore, with additional uncertainty added to the data (6.5\% uncertainty), the curtailment presents some variation to the depth of the curtailment required and the time at which curtailment is required to alleviate the thermal overload. For the $2 \%$ (SE) case the time step, when DG curtailment is required, is the same as the case without any uncertainty. However there is an error in the control depth albeit improved from the previous cases.

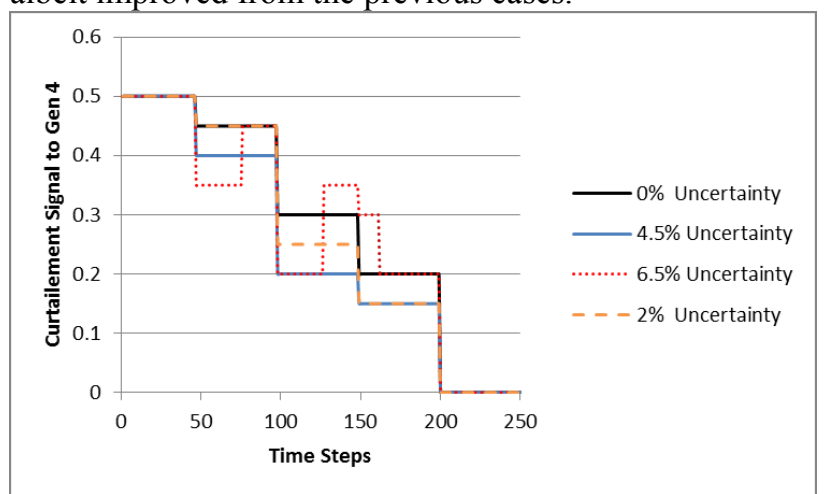

Figure 3 - ANM control signals to Gen 4

In Figure 4 the differences between the control signals (relative to the base case with no uncertainty) sent in presence of uncertainty are shown. These differences relative to the base case with no uncertainty have been considered as the errors in the control action caused by the presence of uncertainty in the input data.

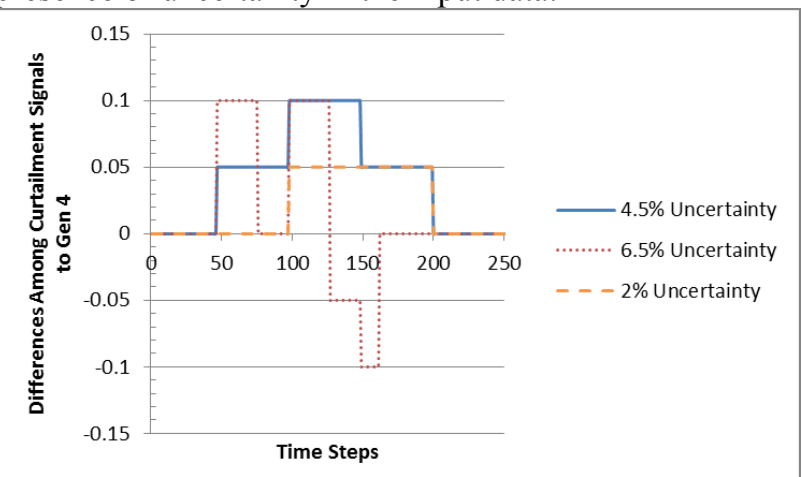

Figure 4 - Difference between control signal values with respect to the case when data had no uncertainty

For the case when using data with an uncertainty of $4.5 \%$ the effect is that the control signal is always overestimated and the average value of the error in the control signal 
calculated over the measurement period is 0.04 , and its variance is $3.4 \%$ of the average. It can be seen in the case of $6.5 \%$ of uncertainty how the control signal could be underestimated. This is an undesirable condition because if the generator is not curtailed enough the integrity of the lines would not be preserved. In this case the average of the error absolute value in the control signal calculated over the measurement period is 0.033 and its variance is $6 \%$ of the average. It is possible to improve the situation by using a SE that provides uncertainty of $2 \%$ and as a consequence reduces the control signal error average calculated over the measurement period to 0.02 (variance: $3 \%$ of the average). A SE reduces the uncertainty of the data provided these are enough data to guarantee the observability of the grid. The SE presented in [9] is used for this simulation with the technique presented in [10] used to find the best position for the minimum set of data that guarantee the observability of the grid and minimize the uncertainty of the estimated data. The technique presented in [10] also enable the calculation of the estimated data uncertainty. With the application of the SE the uncertainty of the majority of the data is reduced from the original $4.5 \%$ to a value between $2 \%$ and $2.5 \%$. Also in this case an overestimation of the necessary control signal is present but is less prevalent than before the SE application. In conclusion, with the growth of the input data uncertainty value from $2 \%$ to $4.5 \%$ it can be observed that the error in the depth of the control action increases. For the $6.5 \%$ of uncertainty it can be noted that the average error does not rise however the variance significantly increases.

\section{CONCLUSION}

This paper has presented the implementation procedure and testing of an ANM function, with regards to data uncertainty in an operational environment, within an experimental microgrid laboratory. The behaviour of the ANM software has been shown when presented with no erroneous data as well as the impact on the control signals when uncertainty is added to the data set. The analysis found that no divergence of the load flow engine was encountered when erroneous measurements, up to $6.5 \%$, were presented to the ANM software. However, with data uncertainty it can be seen that the error, in some situations, is large enough to either move the curtailment to a deeper set point (next domain value for the variable) or not curtail sufficient levels of DG. This suggests that larger gaps between the domain values may have a positive impact on the solution with data uncertainty with the trade-off of curtailing a greater depth of DG. The studies have also highlighted the importance of the reduction in uncertainty through use of a SE. The uncertainty of the input data is reflected in the uncertainty of the final power flow calculation, so the operators taking in account of the uncertainty reduction introduced by a SE can adopt less conservative thermal detection limit to compensate for expected errors.

\section{FUTURE WORK}

Further work in this area would include the evaluation of the data uncertainty value at each cycle of the ANM stage to ascertain potential error mitigation rules for varying errors. This would entail further studies to evaluate the statistical outcomes from this paper for additional case studies over longer time periods. In addition, examining the computational impact of evaluating uncertainty, in a realtime scheme, would be beneficial.

\section{Acknowledgments}

This work has received funding from the European Union Framework 7 program on the basis of Decision No. 912/2009/EC.

\section{REFERENCES}

[1]Currie, R.A.F., et al. Smarter ways to provide grid connections for renewable generators. in Power and Energy Society General Meeting, 2010 IEEE. 2010.

[2]Davidson, E.M., et al. The Use of Constraint Programming for the Autonomous Management of Power Flows. in Intelligent System Applications to Power Systems, 2009. ISAP '09. 15th International Conference on. 2009.

[3]M. J. Dolan, E.M.D., G. W. Ault, K.R.W. Bell, S. D. J. McArthur, Distribution Power Flow Management Utilizing an Online Constraint Programming Method. Smart Grids, IEEE Transaction on.

[4]A. J. Roscoe, A.M., G. M. Burt, and J. R. McDonald, Architecture of a network-in-the-loop environment for characterizing AC power system behaviour. Industrial Electronics, IEEE Transaction on 2010. 57: p. 9.

[5]OpenOPC. [cited 2012 03/10/2012]; Available from: http://openopc.sourceforge.net/.

[6]M. Baran, T.E.M. State Estimation for Real Time Monitoring of Distribution Feeders. in IEEE Power and Energy Society General Meeting. 2009.

[7]R. Singh, B.C.P., R.A. Jabr, Choice of Estimator for Distribution System State Estimation. Generation, Transmission and Distribution, IET, 2009. 3(7): p. 13.

[8]Driesen, J., T. Van Vraenenbroeck, and D. Van Dommelen. The registration of harmonic powers by analogue and digital power meters. in Instrumentation and Measurement Technology Conference, 1997. IMTC/97. Proceedings. Sensing, Processing, Networking., IEEE. 1997.

[9]R. D. Zimmerman, C.E.M.-S., and R. J. Thomas, Matpower: Steady-State Operations, Planning and Analysi Tool for Power System Research. Power Systems, IEEE Transactions on, 2011. 26(1): p. 8.

[10]Alberto Venturi, P.C., Alistair Forbes, Euan Davidson, Andrew. J. Roscoe, Graeme. M.Burt, Member, IEEE, Xin-She Yang and Paul S Wright. The role of accurate measurements within smartgrids. in ISGT Europe 2011. 2011. Manchester. 